\title{
High-pressure synthesis of noble metal hydrides
}

Christian Donnerer, Thomas Scheler, and Eugene Gregoryanz

Citation: The Journal of Chemical Physics 138, 134507 (2013); doi: 10.1063/1.4798640

View online: https://doi.org/10.1063/1.4798640

View Table of Contents: http://aip.scitation.org/toc/jcp/138/13

Published by the American Institute of Physics

\section{Articles you may be interested in}

Metallization and superconductivity of $\mathrm{BeH}_{2}$ under high pressure

The Journal of Chemical Physics 140, 124707 (2014); 10.1063/1.4869145

On the effects of high temperature and high pressure on the hydrogen solubility in rhenium

The Journal of Chemical Physics 135, 214501 (2011); 10.1063/1.3652863

Pressure induced phase transition in $\mathrm{MH}_{2}(\mathrm{M}=\mathrm{V}, \mathrm{Nb})$

The Journal of Chemical Physics 140, 114703 (2014); 10.1063/1.4866179

The metallization and superconductivity of dense hydrogen sulfide

The Journal of Chemical Physics 140, 174712 (2014); 10.1063/1.4874158

Perspective: Crystal structure prediction at high pressures

The Journal of Chemical Physics 140, 040901 (2014); 10.1063/1.4861966

Pressure induced phase transitions in $\mathrm{TiH}_{2}$

Journal of Applied Physics 113, 103512 (2013); 10.1063/1.4795164

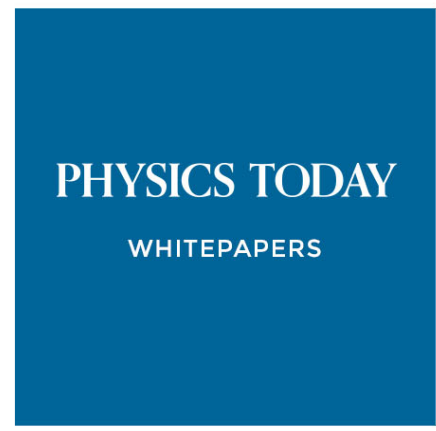

ADVANCED LIGHT CURE ADHESIVES

Take a closer look at what these environmentally friendly adhesive systems can do

\section{READ NOW}

PRESENTED BY Q. MASTERBOND" 


\title{
High-pressure synthesis of noble metal hydrides
}

\author{
Christian Donnerer, Thomas Scheler, and Eugene Gregoryanz ${ }^{\mathrm{a})}$ \\ Centre for Science at Extreme Conditions and School of Physics and Astronomy, The University of Edinburgh, \\ Edinburgh EH9 3JZ, United Kingdom
}

(Received 4 February 2013; accepted 13 March 2013; published online 4 April 2013)

\begin{abstract}
The formation of hydride phases in the noble metals copper, silver, and gold was investigated by in situ $\mathrm{X}$-ray diffraction at high hydrogen pressures. In the case of copper, a novel hexagonal hydride phase, $\mathrm{Cu}_{2} \mathrm{H}$, was synthesised at pressures above $18.6 \mathrm{GPa}$. This compound exhibits an anti-CdI ${ }_{2}^{-}$ type structure, where hydrogen atoms occupy every second layer of octahedral interstitial sites. In contrast to chemically produced $\mathrm{CuH}$, this phase does not show a change in compressibility compared to pure copper. Furthermore, repeated compression (after decomposition of $\mathrm{Cu}_{2} \mathrm{H}$ ) led to the formation of cubic copper hydride at $12.5 \mathrm{GPa}$, a phenomenon attributed to an alteration of the microstructure during dehydrogenation. No hydrides of silver (up to $87 \mathrm{GPa}$ ) or gold (up to $113 \mathrm{GPa}$ ) were found at both room and high temperatures. (C) 2013 American Institute of Physics. [http://dx.doi.org/10.1063/1.4798640]
\end{abstract}

\section{INTRODUCTION}

Despite hydrogen being highly reactive, none of the group-6 to group-11 transition metals react with it at ambient conditions. ${ }^{1-3}$ However, at elevated pressures, the chemical potential of hydrogen rises steeply and reactions, usually leading to the formation of a binary metal hydride, are induced. ${ }^{3-5}$ The group-11 noble metals play an interesting role in this context. While the formation of gold hydride was reported more than 30 years ago, ${ }^{6}$ it has not been confirmed since. In fact, recent $a b$ initio calculations found no evidence for a reaction between gold and hydrogen, due to a large energy barrier preventing the dissociation of hydrogen molecules at the gold surface. ${ }^{7}$ For silver, to the best of our knowledge, no bulk hydride phase has ever been found. Theoretical studies predict that $f c c$-silver hydride stabilises at pressures above $50 \mathrm{GPa}^{8}$

In contrast, copper hydride was one of the very first $d$-metal hydrides to be discovered. It was first synthesised in 1844 by reacting hypophosphorous acid with copper sulphate (known as Wurtz method). ${ }^{9}$ Forming a stoichiometric monohydride, ${ }^{10}$ it adopts a hexagonal wurtzite-type structure (P6 3 mc space group). ${ }^{11}$ This is unusual compared to other transition metal hydrides, many of which exist in NiAs-type or NaCl-type structure. Often, the metal atoms form a closedpacked host lattice in which hydrogen atoms occupy interstitial sites ${ }^{4}$ and participate in metallic bonds. As a result, the presence of hydrogen atoms does not influence the mechanical properties of its host lattice, except for a measurable volume expansion. However, in wurtzite $\mathrm{CuH}$, the bulk modulus was measured to be $72(2) \mathrm{GPa},{ }^{12}$ significantly lower than the bulk modulus of pure copper $(137.4 \mathrm{GPa}) .{ }^{13}$ This can be attributed to the hydrogen-copper bond which has been described as partly covalent and ionic. ${ }^{14,15}$ Chemically produced $\mathrm{CuH}$ is unstable at ambient conditions, but at temperatures

\footnotetext{
a)E-mail: e.gregoryanz@ed.ac.uk
}

below $-5{ }^{\circ} \mathrm{C}$, it can be stored permanently. ${ }^{16}$ At room temperature it decomposes at pressures below 8.4(6) GPa. ${ }^{17}$ Copper hydride is widely used as a reagent for reduction reactions in organic synthesis ${ }^{18}$ and can also be used to obtain extremely reactive catalysts. ${ }^{19}$ Most recently, a facile synthesis of $\mathrm{CuH}$ through sonochemistry was reported. ${ }^{20}$

However, the direct synthesis of hexagonal $\mathrm{CuH}$ from elements under thermodynamic equilibrium (i.e., at high pressures and temperatures) has never been reported. Through Gibbs free energy calculations, based on the decomposition pressure, a formation pressure of $30 \mathrm{GPa}$ was predicted. ${ }^{17} \mathrm{In}$ terestingly, a different form of copper hydride has been synthesised at pressures above $14.4 \mathrm{GPa}$, the only high pressure study of copper hydride formation we are aware of. ${ }^{21}$ This hydride retains the $f c c$ lattice of copper, where hydrogen atoms occupy interstitial sites at a hydrogen-to-metal atomic ratio of $\sim 0.4$. In accordance with the notation for other hydrides, ${ }^{4}$ this cubic phase will be referred to as the $\gamma$-phase of $\mathrm{CuH}$ here. It was suggested that this phase could only be observed in a reaction of hydrogen with dehydrogenated copper, obtained from decomposing hexagonal wurtzite-type $\mathrm{CuH}$ (i.e., copper mono hydride synthesised by the Wurtz method). Conversely, pristine copper foil remained inert and no other hydride phase was observed at pressures up to $20 \mathrm{GPa}^{21}$

In this study, we present results of synchrotron-based powder X-ray diffraction (XRD) experiments on copper, silver, and gold in a dense hydrogen medium. In the copper-hydrogen system, we identified a novel hexagonal hydride phase, distinctly different from wurtzite-type $\mathrm{CuH}$ and $\gamma-\mathrm{Cu}-\mathrm{H}$. This phase formed at $18.6 \mathrm{GPa}$ and remained stable up to $51 \mathrm{GPa}$ in an anti- $\mathrm{CdI}_{2}$ type structure, with a stoichiometric composition of $\mathrm{Cu}_{2} \mathrm{H}$. It decomposes at pressures below $10 \mathrm{GPa}$. On repeated compression a volume expansion in $f c c-\mathrm{Cu}$ was measured, indicating the formation of cubic copper hydride, although with a lower hydrogen content than previously reported. ${ }^{21}$ No hydride phases were observed upon pressurization of silver and gold. 


\section{EXPERIMENTAL DETAILS}

We conducted six independent high-pressure experiments, two each for copper, silver, and gold. Metal samples from commercial thin foils (Alfa Aesar, $10 \mu \mathrm{m}$ thickness, $99.8 \%$ purity) were loaded into symmetrical diamond anvil cells (DACs). Hydrogen was subsequently gas-loaded to pressures between 1600 and 2000 bar and thus also acted as the pressure transmitting medium. The samples were always surrounded by excess hydrogen (monitored visually and by Raman spectroscopy), even at the highest pressures. The gasket material was rhenium, with a hole drilled to a diameter of $\sim 50 \%$ of the culet diameter. Pressure was measured in situ using the revised ruby fluorescence pressure scale. ${ }^{22}$ The diffraction patterns were recorded on MAR345 or MAR555 detectors and integrated using the Fit2D ${ }^{23}$ software.

\section{RESULTS AND DISCUSSION}

In the first experimental run on copper, the metal remained unchanged in its $f c c$ phase ( $F m \overline{3} m$ spacegroup) up to 21.1 GPa. At this pressure, over time, new reflections started to emerge (see Fig. 1). Although the new reflections gained intensity at higher pressures, the $f c c$ signature remained visible up to $51 \mathrm{GPa}$, the highest pressure achieved in this run. Initially, this new phase was indexed in the $P 6_{3} / m m c$ spacegroup ( $h c p$ lattice), an increased volume per formula unit compared to $f c c-\mathrm{Cu}$ indicates the formation of a hydride. The
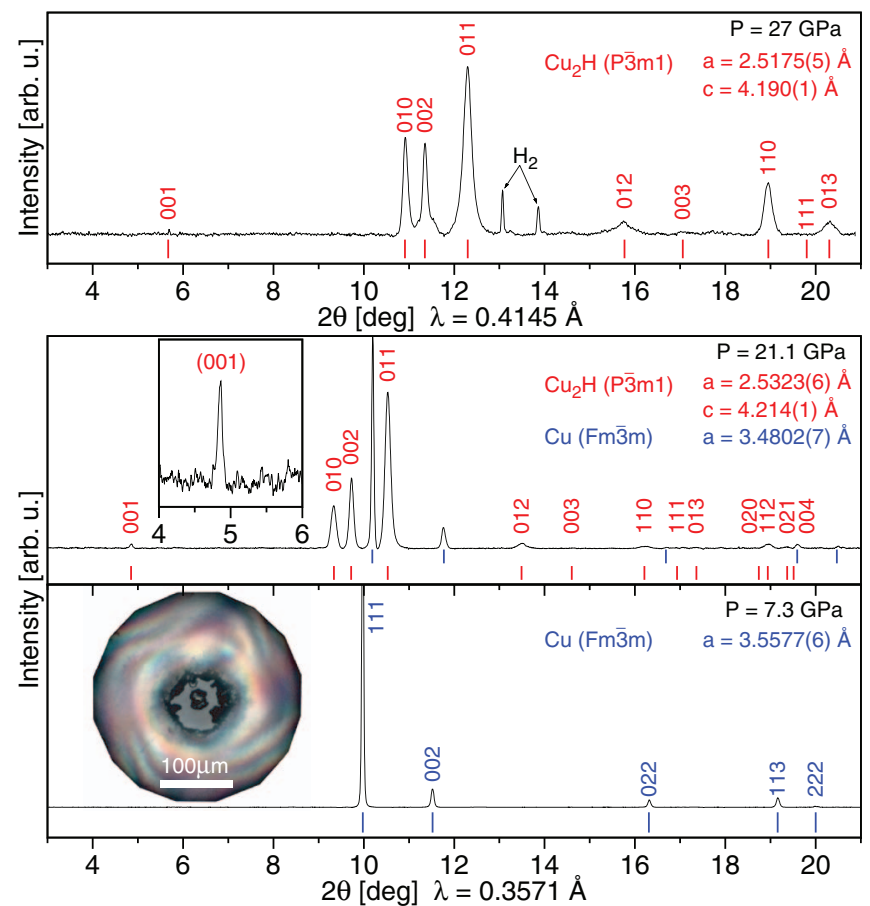

FIG. 1. Integrated XRD spectra of pure $\mathrm{Cu}$ at $7.3 \mathrm{GPa}$ (bottom panel); $\mathrm{Cu}_{2} \mathrm{H}$ along with remaining $\mathrm{Cu}$ at $21.1 \mathrm{GPa}$ (middle panel); the fully converted $\mathrm{Cu}_{2} \mathrm{H}$ along with reflections from excess $\mathrm{H}_{2}$ at $27 \mathrm{GPa}$ (upper panel). Tick marks indicate calculated peak positions of $\mathrm{Cu}$ (blue) in the bottom panel, $\mathrm{Cu}$ (top row, blue), and $\mathrm{Cu}_{2} \mathrm{H}$ (bottom row, red) in the middle panel and $\mathrm{Cu}_{2} \mathrm{H}$ (red) in the upper panel. The inset shows the (001) superstructure peak of the layered anti- $\mathrm{CdI}_{2}$ structure of $\mathrm{Cu}_{2} \mathrm{H}$. The optical micrograph in the bottom panel shows the sample at $50 \mathrm{GPa}$.

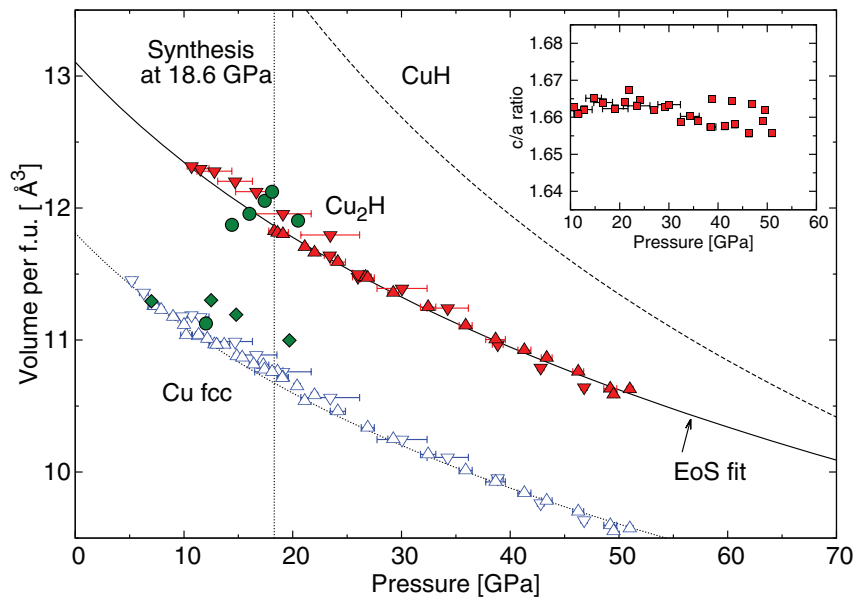

FIG. 2. Equation of state of copper and its hydrides. Blue open triangles denote $f c c-\mathrm{Cu}$, red solid triangles $\epsilon-\mathrm{Cu}_{2} \mathrm{H}$, and green diamonds $\gamma-\mathrm{CuH}_{\sim 0.15}$. Triangles pointing upwards are data points from compression, triangles pointing downwards are from decompression. For comparison, green circles are data points belonging to $\gamma-\mathrm{CuH}_{\sim 0.4}$, taken from Ref. 21 . Dotted and dashed lines are literature equations of state for $f c c-\mathrm{Cu}$ and wurtzite-type $\mathrm{CuH}$, respectively (Refs. 13 and 12). The solid line is a Vinet-type fit to the observed equation of state of $\epsilon-\mathrm{Cu}_{2} \mathrm{H}$. The inset shows the pressure variation of the c/a-ratio for $\epsilon-\mathrm{Cu}_{2} \mathrm{H}$.

new diffraction pattern is also easily distinguishable from rhenium hydride which forms at the inner gasket rim and also exhibits an $h c p$-like structure. ${ }^{24}$ Apart from a smaller unit-cell volume compared to $\mathrm{ReH}$, the $c / a$-ratio of the new hexagonal phase remained at a constant 1.661(3) throughout the experiment (see inset Fig. 2) whereas it is between 1.61 and 1.58 for rhenium hydride. ${ }^{25}$ In the second run, the new phase appeared at 18.6 GPa. In contrast to the first run, the sample converted completely within $2 \mathrm{~h}$, leaving no signal from $\mathrm{fcc}$ $\mathrm{Cu}$. The only difference between the two experiments was the size of the loaded copper sample: The volume of the sample in the second run was an order of magnitude smaller than in the first run. While it is not impossible that with time a larger sample would fully convert, it can be concluded that, even at very high pressures, the penetration depth of hydrogen into bulk copper is limited. A similar behaviour is known from platinum nitride ${ }^{26}$ where only a thin surface layer reacted even at pressures above $50 \mathrm{GPa}$ and temperatures exceeding $2000 \mathrm{~K}$.

(a)

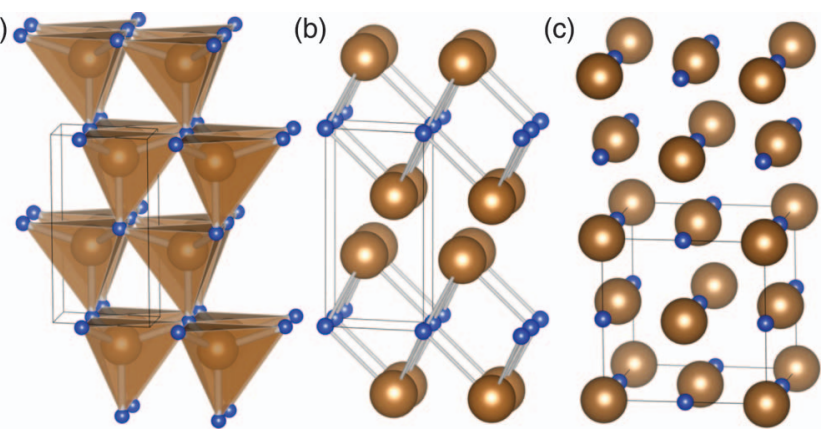

FIG. 3. Ordering of hydrogen (small, blue) and copper (large, copper) atoms showing (a) wurtzite-type $\mathrm{CuH}\left(\mathrm{PG}_{3} / m c\right.$ space group), (b) anti-CdI 2 structure $\epsilon-\mathrm{Cu}_{2} \mathrm{H}(P \overline{3} m 1$ space group, the displacement of copper atoms is exaggerated), and (c) cubic $\gamma-\mathrm{Cu}-\mathrm{H}\left(F m \overline{3} m\right.$ space group) (visualised via VESTA $\left.{ }^{30}\right)$. 
We therefore assume a synthesis pressure of $18.6 \mathrm{GPa}$ for the hexagonal phase. Decomposition occurred at pressures below $10.5 \mathrm{GPa}$, close to the decomposition pressure of wurtzite-type $\mathrm{CuH}\left(8.4(6) \mathrm{GPa}^{17}\right)$. However, the new hexagonal phase is distinctly different: Over the entire experimental pressure range, the volume difference compared to pure $\mathrm{Cu}$ remained between 1.23(1) and 1.05(1) $\AA^{3}$ per atom, much lower than for wurtzite-type $\mathrm{CuH}$ (see Fig. 2). In fact, volume considerations allow to estimate the hydrogen content (see, e.g., Refs. 3, 24, and 27). It has been found empirically that hydrogen atoms occupying the octahedral interstitial sites of an $h c p$ metal expand the lattice by $\sim 2.1 \AA^{3}$ per hydrogen atom. ${ }^{27}$ As the lattice expansion scales linearly with hydrogen content, ${ }^{27}$ it can be inferred that the new hexagonal phase exhibits a $\mathrm{Cu}_{2} \mathrm{H}$ composition. After platinum, ${ }^{28}$ copper is the second $f c c$ metal known to transform into an $h c p$ hydride. Both possess significantly larger $c / a$ ratios (1.66-1.7) than all other $h c p d$-metal hydrides, ${ }^{4}$ providing a signature for this transformation. The hexagonal $\mathrm{Cu}_{2} \mathrm{H}$ phase will be referred to as the $\epsilon$-phase of copper hydride.

A Vinet equation of state was fitted to the data, yielding a zero-pressure volume of $V_{0}=13.10(1) \AA^{3}$ and bulk modulus of $K_{0}=145(5) \mathrm{GPa}\left(K^{\prime}=4.8(3)\right)$ for $\epsilon-\mathrm{Cu}_{2} \mathrm{H}$ (compared to $K_{0}=137.4 \mathrm{GPa}$ for $f c c-\mathrm{Cu}$ ). ${ }^{13}$ This is in agreement with the widely accepted concept that interstitial hydrogen does not influence the mechanical properties of its host metal, but in stark contrast to the mechanical properties of wurtzitetype $\mathrm{CuH}$ (see Table I). This can be attributed to the different bonding of hydrogen atoms in the chemically synthesised hydride, which causes a significant increase in compressibility and volume $\left(V_{0}=16.75 \AA^{3}, K_{0}=72(2) \mathrm{GPa}\right){ }^{12}$ Optical observations of our samples during hydrogenation and at higher pressures did not show a change in appearance. In addition to the absence of changes in the compressibility and the typical volume expansion, this led to the conclusion that the interstitial hydrogen atom donates its electron partly to the $s$ - and $d$-bands of the surrounding metal (as found for many other hydrides ${ }^{3}$ ), such that the hydride retains a metallic character.

It is often assumed that the hydrogen sublattice in metal hydrides with hydrogen:metal ratios below 1 would form a disordered solid. ${ }^{3}$ With a hydrogen:metal ratio of 0.5 in $\epsilon$ $\mathrm{Cu}_{2} \mathrm{H}$, hydrogen atoms would be randomly distributed over the available sites, leaving 50\% vacant. However, in our data, an additional weak reflection can be seen, matching the forbidden (001) reflection of the $h c p$ lattice. Such reflections ( $00 l$, with odd $l$ ) appear when the $c / 2$ translational symmetry is absent in the hexagonal lattice, through atoms being dis-

TABLE I. Equation of state parameters for $\mathrm{Cu}$, wurtzite-type $\mathrm{CuH}$, and hexagonal $\mathrm{Cu}_{2} \mathrm{H}$. The bulk modulus of the chemically obtained $\mathrm{CuH}$ is significantly lower than for pure copper, in contrast to $\mathrm{Cu}_{2} \mathrm{H}$, where the mechanical properties are unchanged.

\begin{tabular}{lcllll}
\hline \hline & Reference & \multicolumn{1}{c}{ EoS type } & $\mathrm{B}_{0}(\mathrm{GPa})$ & $\mathrm{B}_{0}^{\prime}$ & $\mathrm{V}_{0}\left(\AA^{3}\right)$ \\
\hline $\mathrm{Cu}$ & 13 & Birch-Murnaghan & 137.4 & 5.52 & 11.81 \\
$\mathrm{CuH}$ & 12 & Murnaghan & $72(2)$ & $2.7(3)$ & 16.75 \\
$\mathrm{Cu}_{2} \mathrm{H}$ & This work & Vinet & $145(5)$ & $4.8(3)$ & $13.10(1)$ \\
\hline \hline
\end{tabular}

placed from their equilibrium hexagonal positions. This suggests an anti-CdI $\mathrm{I}_{2}$ type superstructure ( $P \overline{3} m 1$ space group) for $\mathrm{Cu}_{2} \mathrm{H}$ : In this structure, instead of occupying random sites, hydrogen atoms fill every second layer of octahedral interstices. This periodic vacancy and filling of sites along the caxis causes the displacement of the metal atoms. The anti$\mathrm{CdI}_{2}$ type structure is also observed in the hydrides of other metals, such as technetium, ${ }^{29}$ cobalt, ${ }^{27}$ and rhenium. ${ }^{24,25} \mathrm{~A}$ comparison between wurtzite, anti-CdI 2 and cubic copper hydride structures is shown in Fig. 3.

The new positions of the metal atoms in the unit-cell are thus given by $\left(\frac{2}{3}, \frac{1}{3}, \frac{1}{4}+\delta\right)$ and $\left(\frac{1}{3}, \frac{2}{3}, \frac{3}{4}-\delta\right)$. The displacement $\delta$ can be determined by comparing calculated with observed intensities. Throughout both experiments, the relative intensity of the (001) reflection, $I_{(001)} / I_{(011)}$, remained at a constant 2.3(3)\%, corresponding to a fractional displacement of $\delta=0.016(2)$, larger than for $\operatorname{CoD}_{0.5}(\delta=0.012)$ and $\mathrm{TcH}_{0.5}(\delta=0.01)$. This may be due to imperfect hydrogen ordering in the latter hydrides: For $\mathrm{CoD}_{0.5}$, neutron diffraction showed that $\sim 4 \%$ of hydrogen atoms occupy $1 \mathrm{~b}$ sites of the lattice, forming a second sublayer that should be vacant in an ideal anti-CdI ${ }_{2}$ structure. ${ }^{27}$ Generally, a lower hydrogen content seems to manifest in smaller displacements, as $\delta=0.007$ for $\mathrm{CoD}_{0.38}$ and 0.009 for $\mathrm{ReH}_{0.36}{ }^{25}$ From the larger displacement in $\mathrm{Cu}_{2} \mathrm{H}$ it can be inferred that the intermediate layers are indeed completely vacant and that the structure is of perfect or nearly perfect anti-CdI 2 -type.

In order to test the hypothesis that $\gamma-\mathrm{Cu}-\mathrm{H}$ only forms with dehydrogenated copper, we decompressed the sample in the $\epsilon$-phase to below decomposition pressure. After the sample fully separated into $\mathrm{Cu}$ and $\mathrm{H}_{2}$, pressure was increased in steps up to $\sim 20 \mathrm{GPa}$. At $12.5 \mathrm{GPa}$, an increase in unit-cell volume of $f c c-\mathrm{Cu}$ was measured, indicating the formation of $\gamma$-Cu-H. Multiple hydride phases have also been observed in other metal-hydrogen systems, such as manganese hydride, which forms both $\gamma$-and $\epsilon$-phases, depending on the temperature during compression. ${ }^{31}$

In the case of $\gamma-\mathrm{Cu}-\mathrm{H}$, the volume difference per atom increased from $0.338(6) \AA^{3}$ at $12.5 \mathrm{GPa}$ to $0.386(6) \AA^{3}$ at $19.7 \mathrm{GPa}$. For $f c c$ metals, hydrogen atoms typically occupy octahedral interstices in the $\gamma$-phase, leading to a volume expansion of $\sim 2.9 \AA^{3}$ per hydrogen atom. ${ }^{32}$ Assuming that this is the case for $\gamma-\mathrm{Cu}-\mathrm{H}$, a hydrogen:metal ratio of $\sim 0.15$ is found. Compared to the results reported by Burtovyy et al. ${ }^{21}$ we find the same synthesis pressure of $12.5 \mathrm{GPa}$, but a smaller volume increase of the host lattice $(3.45(5) \%$ vs $8 \%-11 \%)$ and thus hydrogen content $(\sim 0.15$ compared to $\sim 0.4$ in Ref. 21). If the formation of $\gamma-\mathrm{Cu}-\mathrm{H}$ does indeed depend on the history of the individual copper sample, the difference in hydrogen content in the $\gamma$-phase may be accounted for by different initial hydrogenation conditions: Burtovyy et al. used commercial wurtzite-type copper hydride to prepare their samples, whereas we used pristine copper foil which was hydrogenated under thermodynamic equilibrium. Furthermore, it was speculated ${ }^{21}$ that small crystallites of copper, created through the decomposition of $\mathrm{CuH}$, may show higher reactivity and could thus form the $\gamma$-phase more easily. An alteration of the microstructure during hydrogenation/dehydrogenation cycles is common in metals. This 
process could be more effective in the decomposition of wurtzite-type $\mathrm{CuH}$ than of $\epsilon-\mathrm{Cu}_{2} \mathrm{H}$, leading to favourable conditions for hydrogen uptake in the $\gamma$-phase. A different explanation is that the lower hydrogen content seen in our data originates from slow kinetics, impeding the hydrogenation of the sample. It is not uncommon that kinetic effects play an important role in the formation of isomorphous metal hydrides (i.e., the incorporation of hydrogen into the metal matrix without it undergoing a structural phase transition, see, e.g., rhenium hydride $\left.{ }^{24}\right)$. In the present case, the time difference between crossing synthesis conditions and reaching maximum pressure for the $\gamma$-phase was only $1 \mathrm{~h}$, in contrast to $20 \mathrm{~h}$ between individual data points in the study by Burtovyy et al. At room temperature, higher hydrogen concentrations in the $\gamma$-phase may only be achieved after a certain time.

It is interesting to note that wurtzite-type $\mathrm{CuH}$ could not be synthesised under thermodynamic equilibrium at pressures up to $51 \mathrm{GPa}$. Extrapolation of the equations of state of wurtzite-type $\mathrm{CuH}$ and $\epsilon-\mathrm{Cu}_{2} \mathrm{H}$ shows that both acquire the same volume at approximately $95 \mathrm{GPa}$, due to the larger compressibility of the first hydride. It can be argued that at this pressure the $\epsilon$ phase might undergo a transition to the wurtzite phase.

We compressed silver to $87 \mathrm{GPa}$ and gold to $113 \mathrm{GPa}$ at room temperature without observing any signs of hydrogenation (i.e., volume increase or phase transition). For silver, single-sided laser heating to well above $1000^{\circ} \mathrm{C}$ was applied at $40 \mathrm{GPa}$ while the gold-hydrogen mixture was externally heated to $\sim 300^{\circ} \mathrm{C}$ at pressures up to $45 \mathrm{GPa}$, to no effect in both cases. Figure 4 shows the measured pressure-volume data for silver and gold. No significant deviation from the known equations of state for both metals can be seen. It was predicted that $f c c$-silver hydride stabilises at pressures above $50 \mathrm{GPa},{ }^{8}$ which could not be confirmed. More recently, a theoretical study reported that pressures in excess of $180 \mathrm{GPa}$ might be necessary. ${ }^{7}$ In the same paper, the authors find that the formation of a gold hydride is impeded by a large energy barrier preventing the dissociation of hydrogen molecules at the metal surface. However, hydrogen undergoes a structural

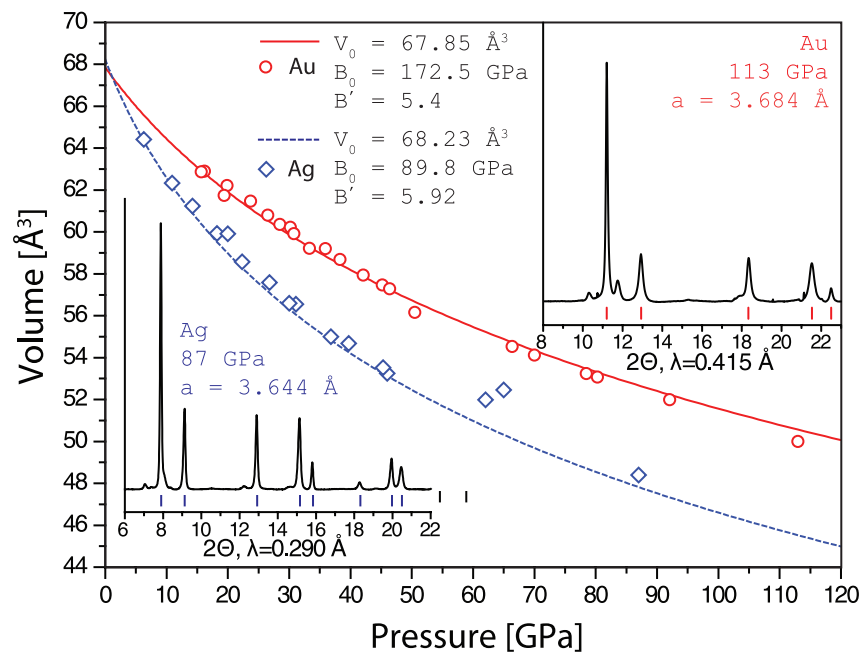

FIG. 4. Equation of state of silver (blue diamonds) and gold (red circles) measured in a hydrogen medium. The lines are literature EoS for $f c c$-silver ${ }^{34}$ and $f c c$-gold. ${ }^{35}$ phase transition into a mixed atomic and molecular state at $220 \mathrm{GPa}$ (phase IV, see Ref. 33). At this point, a direct reaction of gold (and also silver) with hydrogen is very likely.

\section{CONCLUSION}

In conclusion, we synthesised a novel hexagonal copper hydride from elements at pressures above $18.6 \mathrm{GPa}$. This $\epsilon-\mathrm{Cu}_{2} \mathrm{H}$ phase is the third known copper hydride, along with wurtzite-type $\mathrm{CuH}$ and cubic $\gamma-\mathrm{Cu}-\mathrm{H}$. The presence of superstructure peaks allowed to identify the structure as anti- $\mathrm{CdI}_{2}-$ type, where the hydrogen atoms are arranged in layers instead of being randomly oriented. Furthermore, we were able to reproduce the synthesis of $\gamma-\mathrm{Cu}-\mathrm{H}$, albeit with a lower hydrogen concentration. It remains uncertain whether the formation of $\gamma-\mathrm{Cu}-\mathrm{H}$ is promoted by the hydrogenation history or the particular grain size and microstructure of the copper sample. However, it can be concluded that wurtzite-type copper hydride is in fact not the thermodynamically favourable structure of this hydride, despite having the highest hydrogen content and being the easiest to synthesise under non-equilibrium conditions.

\section{ACKNOWLEDGMENTS}

This work is supported by a research grant from the UK Engineering and Physical Sciences Research Council. T.S. gratefully acknowledges the support of the CM-DTC. We thank A. Kleppe from I15, Diamond Light Source, M. Hanfland at ID09A, ESRF, and Z. Konopkova at P02, PETRA-III, for technical support.

${ }^{1}$ F. Lewis, Nature (London) 242, 45 (1973).

${ }^{2}$ B. Hammer and J. Norskov, Nature (London) 376, 238 (1995).

${ }^{3}$ Y. Fukai, The Metal-Hydrogen System: Basic Bulk Properties (Springer, 2005), Vol. 21.

${ }^{4}$ V. E. Antonov, J. Alloys Compd. 330, 110 (2002).

${ }^{5}$ O. Degtyareva, J. Proctor, C. Guillaume, E. Gregoryanz, and M. Hanfland, Solid State Commun. 149, 1583 (2009).

${ }^{6}$ V. E. Antonov, Dokl. Acad. Nauk SSSR 266, 376 (1982).

${ }^{7}$ G. Gao, H. Wang, L. Zhu, and Y. Ma, J. Phys. Chem. C 116, 1995 (2012).

${ }^{8}$ D. Y. Kim, R. H. Scheicher, C. J. Pickard, R. J. Needs, and R. Ahuja, Phys. Rev. Lett. 107, 117002 (2011).

${ }^{9}$ A. Wurtz, C. R. Hebd. Acad. Sci. 18, 702 (1844).

${ }^{10}$ H. Müller and A. Bradley, J. Chem. Soc. 129, 1669 (1926).

${ }^{11} \mathrm{~J}$. Goedkoop and A. Andresen, Acta Crystallogr. 8, 118 (1955).

${ }^{12}$ M. Tkacz, S. Majchrzak, and B. Baranowski, Z. Phys. Chem. 163, 467 (1989).

${ }^{13}$ L. Liu, M. Liu, H. Verbeek, C. Höffner, and G. Will, J. Phys. Chem. Solids 51, 435 (1990)

${ }^{14}$ M. Tkacz and R. Burtovyy, J. Alloys Compd. 404-406, 368 (2005).

${ }^{15}$ S. M. Filipek, J. Adv. Sci. 19, 1 (2007).

${ }^{16}$ N. Fitzsimons, W. Jones, and P. Herley, Catal. Lett. 15, 83 (1992).

${ }^{17}$ M. Tkacz and R. Burtovyy, Solid State Commun. 132, 37 (2004).

${ }^{18}$ M. Semmelhack and R. Stauffer, J. Org. Chem. 40, 3619 (1975).

${ }^{19}$ B. Lipshutz, K. Noson, W. Chrisman, and A. Lower, J. Am. Chem. Soc. 125, 8779 (2003).

${ }^{20}$ P. Hasin and Y. Wu, Chem. Commun. 48, 1302 (2012).

${ }^{21}$ R. Burtovyy and M. Tkacz, Solid State Commun. 131, 169 (2004).

${ }^{22}$ W. Holzapfel, High Press. Res. 25, 87 (2005).

${ }^{23}$ A. P. Hammersley, S. O. Svensson, M. Hanfland, A. N. Fitch, and D. Hausermann, High Press. Res. 14, 235 (1996).

${ }^{24}$ T. Scheler, O. Degtyareva, and E. Gregoryanz, J. Chem. Phys. 135, 214501 (2011).

${ }^{25}$ H. Kawamura, M. Harada, Y. Akahama, and K. Takemura, Solid State Commun. 130, 59 (2004). 
${ }^{26}$ E. Gregoryanz, C. Sanloup, M. Somayazulu, J. Badro, G. Fiquet, H.-K. Mao, and R. J. Hemley, Nat. Mater. 3, 294 (2004).

${ }^{27}$ V. Fedotov, V. Antonov, T. Antonova, E. Bokhenkov, B. Dorner, G. Grosse, and F. Wagner, J. Alloys Compd. 291, 1 (1999).

${ }^{28}$ T. Scheler, O. Degtyareva, M. Marqués, C. Guillaume, J. Proctor, S. Evans, and E. Gregoryanz, Phys. Rev. B 83, 214106 (2011).

${ }^{29}$ V. P. Glazkov, A. V. Irodova, V. A. Somenkov, S. Sh. Shil'shtein, V. E. Antonov, and E. G. Ponyatovskii, Sov. Phys. Solid State 26, 1961 (1984).

${ }^{30}$ K. Momma and F. Izumi, J. Appl. Crystallogr. 41, 653 (2008).
${ }^{31}$ Y. Fukai, T. Haraguchi, H. Shinomiya, and K. Mori, Scr. Mater. 46, 679 (2002).

${ }^{32}$ B. Baranowski, S. Majchrzak, and T. B. Flanagan, J. Phys. F: Met. Phys. 1, 258 (2002).

${ }^{33}$ R. T. Howie, C. L. Guillaume, T. Scheler, A. F. Goncharov, and E. Gregoryanz, Phys. Rev. Lett. 108, 125501 (2012).

${ }^{34}$ Y. Akahama, J. Appl. Phys. 95, 4767 (2004).

${ }^{35}$ A. Dewaele, P. Loubeyre, and M. Mezouar, Phys. Rev. B 70, 094112 (2004). 